\title{
ROLE AND REGULATION OF SUCROSE-PHOSPHATE SYNTHASE IN HIGHER PLANTS
}

\author{
Steven C. Huber ${ }^{1}$ and Joan L. Huber ${ }^{2}$ \\ ${ }^{1}$ United States Department of Agriculture/Agricultural Research Service, and Depart- \\ ments of Crop Science and Botany, North Carolina State University, Raleigh, North \\ Carolina 27695-7631 \\ ${ }^{2}$ Department of Horticultural Science, North Carolina State University, Raleigh, North \\ Carolina 27695-7631
}

KEY WORDS: sucrose synthesis, spinach (Spinacia oleracea L.), maize (Zea mays L.), regulatory protein phosphorylation, protein kinase

\section{ABSTRACT}

Sucrose-phosphate synthase (SPS; E.C. 2.4.1.14) is the plant enzyme thought to play a major role in sucrose biosynthesis. In photosynthetic and nonphotosynthetic tissues, SPS is regulated by metabolites and by reversible protein phosphorylation. In leaves, phosphorylation modulates SPS activity in response to light/dark signals and end-product accumulation. SPS is phosphorylated on multiple seryl residues in vivo, and the major regulatory phosphorylation site involved is Ser158 in spinach leaves and Ser162 in maize leaves. Regulation of the enzymatic activity of SPS appears to involve calcium, metabolites, and novel "coarse" control of the protein phosphatase that activates SPS. Activation of SPS also occurs during osmotic stress of leaf tissue in darkness, which may function to facilitate sucrose formation for osmoregulation. Manipulation of SPS expression in vivo confirms the role of this enzyme in the control of sucrose biosynthesis.

\section{CONTENTS}

INTRODUCTION 


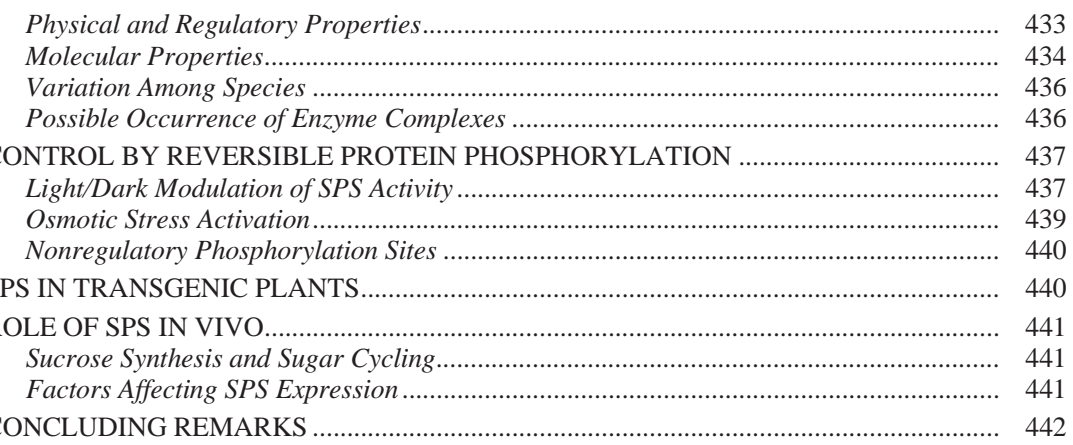

\section{INTRODUCTION}

Sucrose plays a pivotal role in plant growth and development because of its function in translocation and storage, and because of the increasing evidence that sucrose (or some metabolite derived from it) may play a nonnutritive role as a regulator of cellular metabolism, possibly by acting at the level of gene expression (19). It is the nonreducing nature of the sucrose molecule that explains its wide distribution and utilization among higher plants. Trehalose, the only other nonreducing disaccharide found in nature, plays a comparable role in insects and fungi.

Sucrose synthesis can be catalyzed by two distinct enzymes in higher plants: sucrose-phosphate synthase (SPS; EC 2.4.1.14):

UDP-glucose + Fru-6-P $\leftrightarrow$ sucrose-6'-P + UDP + H+ and sucrose synthase (SuSy; EC 2.4.1.13):

UDP-glucose + fructose $\leftrightarrow$ sucrose + UDP $+\mathrm{H}^{+}$.

Although this review focuses on SPS, some comparisons are made with $\mathrm{SuSy}$. Both enzymes are soluble in the cytoplasm and catalyze freely reversible reactions. However, rapid removal of sucrose- 6 '-P by sucrose phosphatase (SPP; EC 3.1.3.24) keeps the cytosolic [sucrose-P] low and thereby renders the SPS reaction essentially irreversible. In fact, recent evidence suggests that SPS and SPP may actually form a complex in vivo (see section on Possible Occurrence of Enzyme Complexes). Thus, sucrose synthesis is generally considered to be catalyzed by SPS (in conjunction with SPP), whereas sucrose breakdown is largely catalyzed by SuSy. Although a given tissue will tend to have an excess of one activity over the other (depending upon whether it is engaged in net sucrose synthesis or breakdown), many tissues have both enzymes, and it is clear that significant "sugar cycling" can occur (discussed below).

A considerable amount has been learned about SPS since its discovery, but still much remains to be learned. For a general discussion of SPS and its role in 
sucrose biosynthesis in leaves, see Stitt et al (36) and Huber et al (16). Since about 1990, several new aspects have been elucidated. With respect to localization, it is now clear that SPS is not confined to photosynthetic tissues but also occurs in nonphotosynthetic tissues that are active in sucrose biosynthesis, e.g. ripening fruits. With respect to mechanisms for control, it is now clear that SPS is controlled $(a)$ at the level of enzyme protein (e.g. leaf development), (b) by allosteric effectors (Glc-6-P and Pi), and (c) by reversible seryl phosphorylation. In addition, the gene encoding SPS has been cloned from several species. As a result, the deduced sequence is available, and cDNA probes can be used to monitor changes in the steady-state pool of SPS mRNA. However, despite the progress made to date, we are just beginning to understand the hierarchy of molecular mechanisms that together control SPS enzymatic activity in vivo. This review focuses on recent developments with SPS.

\section{BIOCHEMICAL AND MOLECULAR PROPERTIES}

\section{Physical and Regulatory Properties}

SPS is a low-abundance protein $(<0.1 \%$ of leaf soluble protein) and is also relatively unstable. Consequently, progress on the purification and characterization of the enzyme has been slow. In addition, there are some apparent differences among species in some of the properties of the enzyme; not all of our information about SPS has been derived from studies of the same enzyme source. Nonetheless, some general statements can be made. It is now generally accepted that substrate saturation profiles for UDP-Glc and Fru-6-P are hyperbolic rather than sigmoidal and that the enzyme from some species can be allosterically activated by Glc-6-P and inhibited by Pi [see Stitt et al (36) for a review]. These effectors have a large effect on the affinities for both substrates, Fru-6-P and UDP-Glc $(29,33)$. Alteration of the affinity for substrates and effectors is also involved in the light modulation of SPS that occurs by reversible protein phosphorylation in some species (38). In general, SPS from nonphotosynthetic tissues (e.g. potato tubers) is regulated by metabolites and by protein phosphorylation in an analogous manner to the enzyme from photosynthetic tissues (29).

The native SPS molecule is likely a dimer of $120-138-\mathrm{kDa}$ subunits (15). Anomalous behavior of SPS on gel-filtration chromatography probably accounts for the larger estimates of molecular mass in some studies. The specific activity of the native spinach enzyme is about $150 \mathrm{IU} / \mathrm{mg}$ protein. In addition, the reaction catalyzed by SPS is clearly reversible. In the most carefully conducted study to date, Lunn \& ap Rees (21) showed that the apparent equilibrium constant $\left(\mathrm{K}_{\mathrm{app}}\right)$ of the pea seed enzyme ranged from 5 to 65

depending upon $\left[\mathrm{Mg}^{2+}\right]$ and $\mathrm{pH}$. Under assumed in vivo conditions, the $\mathrm{K}_{\mathrm{app}}$ 
for SPS has been estimated to be about 10. The calculated mass-action ratio for the SPS reaction in vivo indicates that the reaction is far from equilibrium, presumably because of rapid removal of sucrose-P by SPP.

\section{Molecular Properties}

Cloning of the SPS gene was accomplished first for the enzyme from maize (42) and then for the spinach $(20,35)$, potato (34), sugarbeet (9), and rice (JJ Valdez-Alarco'n, B Jimenez-Moraila \& LR Herrera-Estrella, submitted) enzymes. In general, the N-terminal portions of the $\sim 120-\mathrm{kDa}$ subunit of SPS are highly conserved, and there are also two regions of strong similarity between SPS and SuSy (33). One of the regions that is highly conserved between SPS and SuSy corresponds to residues V176 to S214 of spinach SPS; this region of 39 amino acids exhibits an overall similarity of $64 \%$ between SPS and SuSy (Table 1). Salvucci et al (33) noted that within this highly conserved region there is a stretch of 11 amino acids (D197 to E206 of spinach SPS) that resembles the Gly-rich motif of phosphate-binding domains and thus might be involved in binding of Fru-6-P (to SPS) or UDP-Glc. This stretch contains 10 residues that are identical or similar; the only exception is spinach residue 203, which is a conserved Lys residue in SPS but a conserved Val residue at the analogous position in SuSy. It has been suggested that the function of the basic residue in SPS may influence the selectivity for the negatively charged Fru-6-P rather than the neutral Fru molecule.

The portion of the primary sequence in the vicinity of the uridine moiety of the substrate molecule UDP-Glc has been determined by photoaffinity labeling of a recombinant spinach SPS fragment using $\left[\beta-{ }^{32} \mathrm{P}\right] 5-\mathrm{N}_{3}$ UDP-Glc (32). It was determined that the 5 position of the uridine ring was proximal to the primary sequence Q227 to E239. Note that this sequence is reasonably close to the residues thought to be involved in binding of the other substrate, Fru-6-P (D197 to E206). The uridine-binding region of the SPS molecule is highly conserved among spinach, maize, and potato, but there is relatively little homology with SuSy (33). This is perhaps not surprising because there are no recognized consensus binding motifs for UDP-Glc or other nucleotide diphosphate sugars.

The second region of strong similarity between SPS and SuSy is located toward the C-terminus of the SuSy sequence (residues D587 to P631 of spinach SPS). Of the 44 residues within this region of the SPS molecule, 25 residues are identical and 8 are similar, for an overall similarity of $75 \%$. However, the function of this portion of the molecule is not known.

Another important domain of the SPS molecule that remains to be identified is the effector site involved in Glc-6-P and Pi binding. None of the specific amino acid residues at the effector site has yet been identified. However, the allosteric site contains essential and accessible sulfhydryl group(s), 


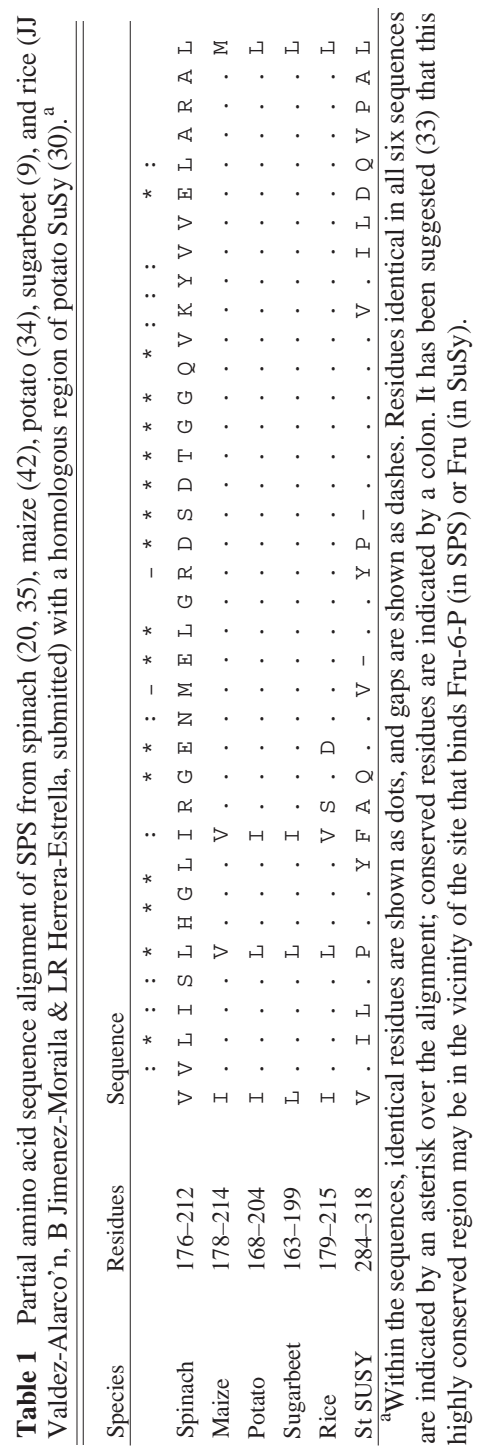


whereas the catalytic site does not (2). Comparison of the SPS sequences available to date indicates that there are 10 conserved cysteine residues. Presumably, one or more of these is at the effector site. It is not known whether all the sulfhydryl groups in the molecule are reduced; however, it is known that there are no intersubunit disulfide bonds (J Huber, unpublished data, 1995).

\section{Variation Among Species}

There appear to be significant quantitative differences among species in the regulatory properties of SPS in vitro, i.e. the extent of Glc-6-P activation and Pi inhibition $(1,13)$. There are also differences in the modulation of SPS in vivo. Some species exhibit a marked light activation of SPS (designated as class I and class II species) (13), whereas others do not (class III species). The distinctions among the three classes of plants are quantitative rather than qualitative in nature. For example, our original studies of soybean (a class III species) involved cultivars of maturity group VII, e.g. "Ransom." Although soybean Ransom plants exhibited little, if any, light activation of SPS in vivo, we have recently observed that soybean cultivars of maturity group OOO, e.g. "Maple Presto," show significant light activation (S Huber, unpublished data, 1994). Similarly, we have observed differences among Nicotiana species and among cultivars of Nicotiana tabacum (S Huber, unpublished data). However, with $N$. tabacum, even when light activation occurs, it is subtle relative to species of classes I and II. Nonetheless, these results suggest that the requisite interconversion enzymes [SPS-kinase (SPSk) and SPS-protein phosphatase (SPS-PP)] may be present at some level in all species.

Further support for this notion has recently been obtained from studies of transgenic tobacco plants expressing the maize SPS gene (18). In control plants, there was a small but significant $(\sim 30 \%$ increase in the light compared with dark) activation of SPS assayed under selective conditions (limiting substrates plus allosteric inhibitor, $\mathrm{Pi}$ ). In transgenic tobacco plants expressing the maize SPS gene, $\mathrm{V}_{\max }$ activity of SPS was increased about 2.5 -fold as a result of expression of the transgene, and the maize enzyme was light activated in a very pronounced manner ( $150 \%$ increase in the light). These results are noteworthy because maize SPS expressed in transgenic tomato plants shows relatively little light modulation $(4,18)$. The basis for the lack of modulation in this case is not clear but may involve slight differences in quaternary structure, which results in the phosphorylation site becoming less accessible to the endogenous protein kinases.

\section{Possible Occurrence of Enzyme Complexes}

There is increasing evidence from other systems that soluble enzymes often occur as complexes with other related enzymes. In the case of SPS, there is evidence for an association with SPSk (12), which may facilitate the phospho- 
rylation of this low-abundance protein. Recent evidence also shows that SPS and SPP may form a complex in vitro. The primary observation is that SPS activity in vitro is reversibly reduced by removal of SPP during purification (GL Salerno, E Echeverria, HG Pontis, submitted); efficient removal of inhibitory sucrose-6'-P via an enzyme complex is speculated to be necessary for maximal SPS activity. It will be interesting to determine whether phosphorylation of SPS (at either regulatory or nonregulatory sites) affects the interaction with SPP.

\section{CONTROL BY REVERSIBLE PROTEIN PHOSPHORYLATION}

\section{Light/Dark Modulation of SPS Activity}

REGULATORY PHOSPHORYLATION SITE The major (if not sole) regulatory phosphorylation site of spinach SPS has been identified (23) as Ser158 (Table 2). Phosphorylation of Ser158 is both necessary and sufficient for the inactivation of SPS in vitro. Additional lines of evidence consistent with the assignment include: (a) labeling of the tryptic phosphopeptide containing Ser158 [previously designated phosphoprotein 7 (11)] in situ correlates with inactivation of SPS; (b) a synthetic peptide based on the phosphorylation site sequence is a good substrate for SPSk in vitro and competes with native SPS for phosphorylation/inactivation (22); (c) labeling of Ser158 in situ occurs more rapidly than other (nonregulatory) phosphorylation sites on SPS and reflects different turnover rates (17); and (d) polyclonal antibodies generated against the phosphorylation site sequence preferentially recognize and immunoprecipitate highly activated dephospho-SPS as opposed to inactivated phospho-SPS (40).

Although the regulatory phosphorylation sequence of spinach SPS is not conserved exactly, all sequences available to date contain a homologous seryl

Table 2 Amino acid sequences surrounding the putative regulatory phosphorylation site of SPS. ${ }^{\text {a }}$

\begin{tabular}{lcllllllllllllllllll}
\hline \hline Species & Residues & \multicolumn{1}{l}{ Sequence } & : & & : & : & & $*$ & & $*$ & & & & & : \\
Spinach & $150-162$ & K & G & R & M & R & R & I & S & $\underline{\mathbf{S}}$ & V & E & M & M \\
Potato & $142-154$ & R & G & R & L & P & R & I & S & $\underline{\mathbf{S}}$ & V & E & T & M \\
Sugarbeet & $137-149$ & R & P & R & L & P & R & I & N & $\underline{\mathbf{S}}$ & L & D & A & M \\
Maize & $154-166$ & K & K & K & F & Q & R & N & F & $\underline{\mathbf{S}}$ & D & V & T & L \\
Rice & $154-166$ & K & K & K & F & Q & R & N & F & $\underline{\mathbf{S}}$ & E & L & T & V \\
& & -8 & & -6 & & & -3 & & & 0 & & & & 4 \\
\hline
\end{tabular}

${ }^{\mathrm{a}}$ Residues identical in all five sequences are indicated by an asterisk over the alignment; conserved residues are indicated by a colon. Residues are numbered relative to the phosphorylated Ser at position 0 . Spinach $(20,35)$, maize (42), potato (34), sugarbeet (9), and rice (JJ Valdez-Alarco'n, B Jimenez-Moraila \& LR Herrera-Estrella, submitted). 
residue (Table 2). Evidence consistent with phosphorylation of Ser162 in maize SPS has been obtained in studies of maize leaves as well as transgenic tobacco expressing the maize SPS gene (18). It remains to be determined whether the homologous Ser residue in the other species is phosphorylated and is of regulatory significance, but it seems quite likely. It is important to note that several of the residues surrounding the (putative) phosphorylation site are also conserved among the five species. In particular, there are basic residues at $\mathrm{P}-3, \mathrm{P}-6$, and $\mathrm{P}-8$ (numbering relative to the Ser at position 0 ) and hydrophobic residues at $\mathrm{P}-5$ and $\mathrm{P}+4$ (Table 2). At least several of these conserved residues appear to be important for recognition by protein kinase.

SPS-KINASE Partially purified spinach leaf SPS contains a copurifying protein kinase that can phosphorylate and inactivate SPS with $\left[\gamma_{-}^{32} \mathrm{P}\right] \mathrm{ATP}(12)$. In vitro, approximately $75-85 \%$ of the ${ }^{32} \mathrm{P}$ is incorporated into Ser158, the major regulatory phosphorylation site. Using a synthetic peptide based on the phosphorylation site sequence, two protein kinases with apparent molecular masses of 45 and $150 \mathrm{kDa}$ were resolved chromatographically from spinach leaves (22). The smaller kinase (designated peak I) is most likely a monomer, whereas the larger kinase (peak III) has a subunit molecular mass of $\sim 65 \mathrm{kDa}$. An important distinction between the two kinases is that the peak I enzyme is strictly $\mathrm{Ca}^{2+}$ dependent, whereas the peak III enzyme, which tends to copurify with SPS, is $\mathrm{Ca}^{2+}$ independent. The substrate specificity of both kinases has been characterized in vitro using synthetic peptide analogs. The major recognition elements consist of basic residues at P-6 and P-3 (24) and a hydrophobic residue at P-5 (S Huber \& D Toroser, unpublished data, 1995). These residues are also conserved among species (Table 2).

Studies with maize leaf SPSk have identified a single form of the enzyme, and there is a clear requirement for peptide substrates with basic residues at $\mathrm{P}-3 / \mathrm{P}-6$ and a hydrophobic residue at P-5 (R McMichael \& S Huber, unpublished data, 1994). Maize leaf SPSk is also strictly $\mathrm{Ca}^{2+}$ dependent (18). These observations raise the intriguing possibility that cytosolic $\left[\mathrm{Ca}^{2+}\right]$ may regulate sucrose biosynthesis, at least in some species. There is evidence that cytosolic $\left[\mathrm{Ca}^{2+}\right]$ is reduced in the light relative to the dark (26). These changes in cytosolic $\left[\mathrm{Ca}^{2+}\right]$ could contribute to the light activation of SPS in vivo (Figure 1 ). Another factor that may be important in vivo is Glc-6-P, which is not only an allosteric activator of SPS but also an inhibitor of SPSk per se (24).

SPS-PROTEIN PHOSPHATASE Phospho-SPS is dephosphorylated/activated by a type 2A protein phosphatase (SPS-PP) that is inhibited by Pi (13). In spinach, there is a distinct light activation of SPS-PP that involves an increase in total extractable activity as well as a decrease in sensitivity to Pi inhibition (41). The light activation of SPS-PP can be blocked by pretreatment of leaves with 


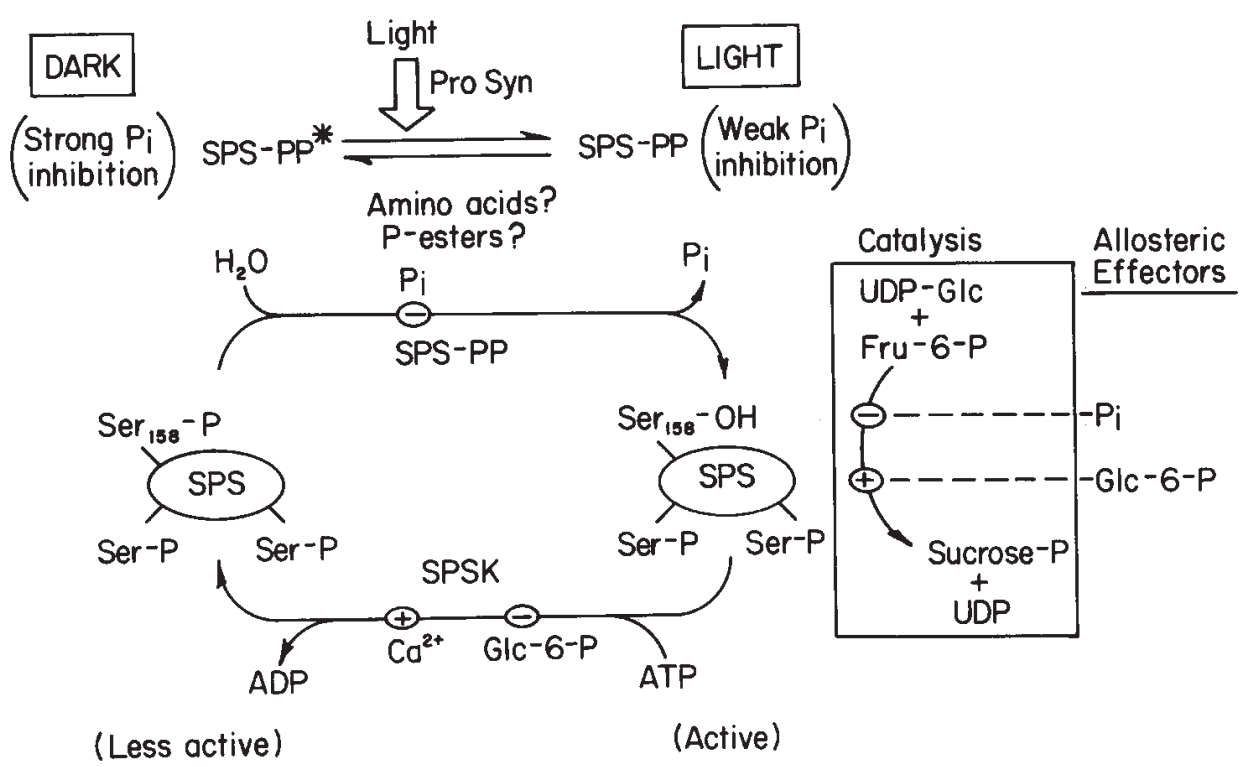

Figure 1 Schematic representation of the regulation of spinach leaf SPS by reversible seryl phosphorylation. Multisite phosphorylation and the identification of the major regulatory site as Ser158 are indicated. An increase in Glc-6-P and a decrease in Pi, as might occur during a dark-to-light transition, would favor dephosphorylation/activation of SPS and would also increase catalytic activity as a result of allosteric regulation. Another important factor may be light modulation of the regulatory properties of SPS-PP, and changes in cytosolic $\left[\mathrm{Ca}^{2+}\right]$. Adapted from Reference 13.

cycloheximide (CHX), which suggests a role for cytoplasmic protein synthesis. However, the molecular basis for the light activation remains unclear; it could result from either a covalent modification of existing protein or the synthesis of a target/regulatory subunit or modifying enzyme. Regardless of the mechanism, the light modulation of SPS-PP and its regulation by Pi are thought to play an important role in the activation of SPS after a dark-to-light transition (Figure 1). Other potential effectors of SPS-PP include a variety of P-esters (41) and amino acids (14). The inhibition by amino acids may play an important role in feedback regulation of sucrose synthesis.

\section{Osmotic Stress Activation}

Activation of SPS, assayed under selective conditions, occurs in spinach leaves (28) and potato tubers (29) incubated in hyperosmotic solutions of mannitol or sorbitol. The simplest explanation, dephosphorylation of the regu- 
latory site in response to the stress, seems not to be the case. Rather, it appears that a unique site(s) on spinach SPS is phosphorylated during osmotic stress that can partially antagonize the inhibitory effect of phosphorylation of the regulatory site (Ser158 in spinach). Control of this process may occur at the level of gene expression (14). Indeed, stress-induced protein kinases have been demonstrated (10). Whether a stress-induced kinase and the identity of the putative novel phosphorylation site are involved remains to be established.

\section{Nonregulatory Phosphorylation Sites}

Spinach leaf SPS appears to be phosphorylated on multiple seryl residues in vivo. Apart from the regulatory site (Ser158 in spinach), the phosphorylation status of the other sites remains relatively constant during light/dark transitions, i.e. the sites may be constitutively phosphorylated. We have tentatively identified two of the nonregulatory sites (17) and are in the process of identifying endogenous protein kinases that might phosphorylate these residues.

\section{SPS IN TRANSGENIC PLANTS}

Increased activity of SPS in leaves has been achieved in several species by overexpression of the gene encoding SPS in transgenic plants. Transgenic tomato plants expressing the maize SPS gene had elevated leaf SPS, and the maize enzyme was unregulated with respect to normal light/dark modulation $(4,5,42)$. The enhanced SPS activity was associated with an increased lightand $\mathrm{CO}_{2}$-saturated rate of photosynthesis and, under ambient conditions, with increased ratios of sucrose to starch in leaves (4) and increased partitioning of fixed-C into sucrose (25). Overall growth of the transgenic tomato plants was not increased when the transgene expression was leaf-specific, i.e. expressed from the Rubisco small subunit promoter. However, recent results suggest that growth enhancement may occur when SPS expression is constitutive (i.e. $35 \mathrm{~S}-\mathrm{CaMV}$ promoter) and occurs in both photosynthetic and nonphotosynthetic tissues (3). Micallef et al (25) also found that vegetative growth of transgenic tomato plants (expressing the maize SPS gene driven by the Rubisco SSU promoter) was not increased but noted that reproductive development was enhanced. Total fruit number was increased, the fruit matured earlier, and there was a substantial increase in total fruit dry weight (25). More work needs to be done on the growth response itself and to determine the basis for the enhancement when it is observed, but it is speculated that SPS, by affecting tissue [sucrose] might influence flowering at least in some species. Another intriguing observation by Micallef et al (25) is that SPS transformants did not exhibit the normal acclimation response of leaf photosynthesis to high $\mathrm{CO}_{2}$. Thus, when grown and measured at high $\mathrm{CO}_{2}$, the SPS transformants had 
higher rates of photosynthesis per unit leaf area compared with the control plants.

Spinach SPS has also been expressed in transgenic tobacco and potato plants. However, despite an increase in SPS protein, the additional enzyme was downregulated, apparently by phosphorylation, such that metabolism was not affected (37). In order to effectively upregulate SPS activity and sucrose biosynthesis, it may be necessary to produce plants expressing a genetically modified SPS protein, e.g. with the regulatory phosphorylation site removed.

A substantial reduction in SPS activity in potato leaves and tubers has been achieved with an SPS-antisense construct under control of the 35S-CaMV promoter (7). As a result of the antisense inhibition, sucrose synthesis in leaves was reduced, and starch and amino acid synthesis was increased. A flux control coefficient for SPS was estimated to be $0.30-0.45$ in potato leaves. In tubers, the resynthesis of sucrose from starch was reduced in the SPS-antisense transformants. Overall, the results strongly support the notion that SPS is one of the important control points in sucrose biosynthesis.

\section{ROLE OF SPS IN VIVO}

\section{Sucrose Synthesis and Sugar Cycling}

In addition to the well-recognized role of SPS in sucrose biosynthesis in source leaves, it is becoming clear that some sucrose synthesis occurs even in heterotrophic cells that are engaged in net sucrose degradation. Significant turnover of the endogenous sucrose pool has been identified in a variety of tissues, including potato tubers (7) and germinating Ricinus cotyledons (8). Turnover of sucrose is thought to involve a futile cycle of simultaneous synthesis (by SPS and SuSy) and cleavage (by SuSy). Thus, relatively small changes in unidirectional fluxes can occur and produce much larger changes in net flux through the sucrose pool, without large changes in metabolites (7). Changes in the activation state of SPS, presumably as a result of protein phosphorylation, have been shown to contribute to changes in net flux through the sucrose pool in Ricinus cotyledons (8) and potato tubers (7).

\section{Factors Affecting SPS Expression}

Expression of SPS mRNA and enzyme protein is controlled developmentally, e.g. during leaf development (20), and in mature leaves by a variety of factors, including irradiance (20) and N-nutrition (J Huber, unpublished data, 1994). The responses of SPS to changes in irradiance illustrate the integration of mechanisms for control of SPS activity. Transfer of spinach plants grown at low irradiance to high irradiance results in a rapid increase in net photosynthesis and flux of $\mathrm{C}$ into sucrose. Within $3 \mathrm{~h}$ of transfer, SPS protein (and $\mathrm{V}_{\max }$ 
activity) remain constant, but activation state of the enzyme is increased presumably by dephosphorylation of Ser158 (15). After longer periods of time at the higher irradiance, there is a gradual increase in SPS protein and mRNA (20). Thus, regulation of enzyme activity by covalent modification and control of SPS gene expression function in an integrated manner to provide short- and long-term control, respectively.

SPS gene expression also responds to sugars. Provision of Glc to excised sugarbeet or potato leaves strongly increased the steady state level of SPS mRNA, whereas exogenous sucrose slightly repressed expression [at least in sugarbeet (9)]. A similar response is seen in potato tubers when starch synthesis is inhibited by antisense repression of ADP-Glc pyrophosphorylase. The transgenic potato tubers accumulated soluble sugars (sucrose and glucose), and there was a tremendous increase in the steady state level of SPS mRNA (27). SPS activity, measured under selective assay conditions, was also increased relative to wild-type tubers (7), but the basis for the increased activity was not determined. The results suggest that hexose sugars, or some related metabolite(s), might be involved in the control of expression of SPS as well as other genes. It is important to note that the sugar effects on SPS expression have been identified both when exogenous sugars are provided (9) and when endogenous sugars are manipulated genetically (27).

\section{CONCLUDING REMARKS}

With the cloning of the SPS gene from five species, we are beginning to better understand the SPS molecule with preliminary identification of important domains such as substrate binding sites and phosphorylation sites. In addition to its role in source leaves, SPS is also significant in sink tissues where a futile cycle of simultaneous degradation and resynthesis occurs in a wide range of tissues. Manipulation of SPS activity is now possible and holds promise for impacting on plant growth and resource allocation. SPS is clearly an important factor regulating sucrose biosynthesis, but it is important to recognize that it is not the only factor, and in addition, changes in SPS protein level are often compensated for by adjustments in the activation state of the enzyme as a result of phosphorylation/dephosphorylation. Consequently, future transformation studies need to consider production of plants with SPS protein modified in terms of phosphorylation control.

\section{ACKNOWLEDGMENTS}

We thank colleagues for sharing unpublished manuscripts and ideas, especially Mike Salvucci, Christine Foyer, Mark Stitt, Luis Herrera-Estrella, and Lothar Willmitzer, and other members of the laboratory for discussions. Financial support from the US Department of Agriculture/Agricultural Research 
Service, the USDA National Research Initiative Competitive Grant Program, and the Department of Energy is gratefully acknowledged.

Any Annual Review chapter, as well as any article cited in an Annual Review chapter, may be purchased from the Annual Reviews Preprints and Reprints service. 1-800-347-8007; 415-259-5017; email: arpr@class.org

\section{Literature Cited}

1. Crafts-Brandner SJ, Salvucci ME. 1989. Species and environmental variations in the effect of inorganic phosphate on sucrosephosphate synthase activity. Plant Physiol. 91:469-72

2. Doehlert DC, Huber SC. 1985. The role of sulfhydryl groups in the regulation of spinach leaf sucrose-phosphate synthase. Biochim. Biophys. Acta 830:267-73

3. Foyer CH, Galtier N, Quick P. 1994. Modifications in carbon assimilation, carbon partitioning and total biomass as a result of over-expression of sucrose phosphate synthase in transgenic tomato plants. Plant Physiol. 105:S23

4. Galtier N, Foyer CH, Huber JLA, Voelker TA, Huber SC. 1993. Effects of elevated sucrose-phosphate synthase activity on photosynthesis, assimilate partitioning and growth in tomato (Lycopersicon esculentum var. UC 82B). Plant Physiol. 101: 535-43

5. Galtier N, Foyer CH, Murchie E, Alred R, Quick P, et al. 1995. Effects of light and atmospheric carbon dioxide enrichment on photosynthesis and carbon partitioning in the leaves of tomato (Lycopersicon esculentum L.) plants over-expressing sucrosephosphate synthase. J. Exp. Bot. 46: 1335-44

6. Deleted in proof

7. Geigenberger P, Krause K-P, Hill LM, Reimholz R, MacRae E, et al. 1995. The regulation of sucrose synthesis in leaves and tuber of potato plants. In Sucrose Metabolism, Biochemistry, Physiology and Molecular Biology, ed. H Pontis, G Salerno, E Echeverria. Rockville, MD: Am. Soc. Plant Physiol.

8. Geigenberger P, Stitt M. 1991. A "futile" cycle of sucrose synthesis and degradation is involved in regulating partitioning between sucrose, starch and respiration in cotyledons of germinating Ricinus communis L. seedlings when phloem transport is inhibited. Planta 185:81-90

9. Hesse H, Sonnewald U, Willmitzer L. 1995. Cloning and expression analysis of sucrose-phosphate synthase from sugar beet (Beta vulgaris). Mol. Gen. Genet. 247: 515-20

10. Holappa LD, Walker-Simmons MK. 1995. The wheat abscisic acid-responsive protein kinase mRNA, PKABA1, is up-regulated by dehydration, cold temperature, and osmotic stress. Plant Physiol. 108:1203-10

11. Huber JLA, Huber SC. 1992. Site specific serine phosphorylation of spinach leaf sucrose-phosphate synthase. Biochem. J. 283:877-82

12. Huber SC, Huber JL. 1991. In vitro phosphorylation and inactivation of spinach leaf sucrose-phosphate synthase by an endogenous protein kinase. Biochim. Biophys. Acta 1091:393-400

13. Huber SC, Huber JLA. 1992. Role of sucrose-phosphate synthase in sucrose metabolism in leaves. Plant Physiol. 99: 1275-78

14. Huber SC, Huber JLA, McMichael RW Jr. 1993. The regulation of sucrose synthesis in leaves. In Carbon Partitioning Within and Between Organisms, ed. CJ Pollock, JF Farrar, AJ Gordon, pp. 1-26. Oxford: BIOS Sci.

15. Huber SC, Huber JL, McMichael RW Jr. 1994. Control of plant enzyme activity by reversible protein phosphorylation. Int. Rev. Cytol. 149:47-98

16. Huber SC, Huber JL, Pharr DM. 1993. Assimilate partitioning and utilization in source and sink tissues. In International Crop Science, ed. DR Buxton, I:761-77. Madison, WI: Crop Sci. Soc. Am.

17. Huber SC, McMichael RW Jr, Bachmann M, Huber JL, Shannon JC, et al. 1995. Regulation of leaf sucrose-phosphate synthase and nitrate reductase by reversible protein phosphorylation. In Protein Phosphorylation in Plants, ed. PR Shewry. Oxford: Oxford Univ. Press. In press

18. Huber SC, McMichael RW Jr, Huber JL, Bachmann M, Yamamoto YT, Conkling MA. 1995. Light regulation of sucrose synthesis: role of protein phosphorylation and possible involvement of cytosolic $\left[\mathrm{Ca}^{2+}\right]$. In Carbon Partitioning and Source-Sink Interactions in Plants, ed. MA Madore, W 


\section{HUBER \& HUBER}

Lucas, pp. 35-44. Rockville, MD: Am Soc. Plant Physiol.

19. Jang J-C, Sheen J. 1994. Sugar sensing in higher plants. Plant Cell 6:1665-79

20. Klein RR, Crafts-Brandner SJ, Salvucci ME. 1993. Cloning and developmental expression of the sucrose-phosphate-synthase gene from spinach. Planta 190: 498-510

21. Lunn JE, ap Rees T. 1990. Apparent equilibrium constant and mass-action ratio for sucrose-phosphate synthase in seeds of Pisum sativum. Biochem. J. 267:739-43

22. McMichael RW Jr, Bachmann M, Huber SC. 1995. Spinach leaf sucrose-phosphate synthase and nitrate reductase are phosphorylated/inactivated by multiple protein $\mathrm{ki}-$ nases in vitro. Plant Physiol. 108:1077-82

23. McMichael RW Jr, Klein RR, Salvucci ME Huber SC. 1993. Identification of the major regulatory phosphorylation site in sucrosephosphate synthase. Arch. Biochem. Biophys. 307:248-52

24. McMichael RW Jr, Kochansky J, Klein RR, Huber SC. 1995. Characterization of the substrate specificity of sucrose-phosphate synthase protein kinase. Arch. Biochem. Biophys. 321:71-75

25. Micallef BJ, Haskin KA, Vanderveer PJ, Roth K-S, Shewmaker CK, Sharkey TD. 1995. Altered photosynthesis, flowering and fruiting in transgenic tomato plants that have an increased capacity for sucrose synthesis. Planta 196:327-34

26. Miller AJ, Sanders D. 1987. Depletion of cytosolic free calcium induced by photosynthesis. Nature 326:397-400

27. Müller-Röber BT, Sonnewald U, Willmitzer L. 1992. Inhibition of ADP-glucose pyrophosphorylase leads to sugar storing tubers and influences tuber formation and expression of tuber storage protein genes. EMBO J. 11:1229-38

28. Quick P, Siegl G, Neuhaus HE, Feil R, Stitt M. 1989. Short term water stress leads to a stimulation of sucrose synthesis by activating sucrose phosphate-synthase. Planta 177:536-46

29. Reimholz R, Geigenberger P, Stitt M. 1994. Sucrose phosphate synthase is regulated via metabolites and protein phosphorylation in potato tubers, in a manner analogous to the enzyme in leaves. Planta 1992: 480-88

30. Salanoubat M, Belliard G. 1987. Molecular cloning and sequencing of sucrose synthase
cDNA from potato (Solanum tuberosum L.): preliminary characterization of sucrose synthase mRNA distribution. Gene 60 47-56

31. Deleted in proof

32. Salvucci ME, Klein RR. 1993. Identification of the uridine-binding domain of sucrose-phosphate synthase: expression of a region of the protein that photoaffinity labels with 5-azidouridine diphosphate-glucose. Plant Physiol. 102:529-36

33. Salvucci ME, van de Loo FJ, Klein RR. 1995. The structure of sucrose-phosphate synthase. In Sucrose Metabolism, Biochemistry, Physiology and Molecular Biology, ed. HG Pontis, GL Salerno, E Echeverria. Rockville, MD: Am. Soc. Plant Physiol.

34. Sonnewald U, Basner A. 1993. EMBL Data Library, Accession No. S34172

35. Sonnewald U, Quick WP, MacRae E, Krause KP, Stitt M. 1993. Purification, cloning and expression of spinach leaf sucrose-phosphate synthase in E. coli. Planta 189:174-81

36. Stitt M, Huber SC, Kerr P. 1987. Control of photosynthetic sucrose formation. In Biochemistry of Plants, ed. MD Hatch, NK Boardman, 8:327-409. New York: Academic

37. Stitt M, Sonnewald U. 1995. Regulation of metabolism in transgenic plants. Annu. Rev Plant Physiol. Plant Mol. Biol. 46:341-68

38. Stitt M, Wilke I, Feil R, Heldt HW. 1988. Coarse control of sucrose-phosphate synthase in leaves: alterations of the kinetic properties in response to the rate of photosynthesis and the accumulation of sucrose. Planta 174:217-30

39. Deleted in proof

40. Weiner H. 1995. Antibodies that distinguish between the serine-158 phospho- and dephospho-form of spinach leaf sucrosephosphate synthase. Plant Physiol. 108: 219-25

41. Weiner H, Weiner H, Stitt M. 1993. Sucrose-phosphate synthase phosphatase, a type $2 \mathrm{~A}$ protein phosphatase, changes its sensitivity towards inhibition by inorganic phosphate in spinach leaves. FEBS Lett. 333:159-64

42. Worrell AC, Bruneau J-M, Summerfelt K, Boersig M, Voelker TA. 1991. Expression of a maize sucrose phosphate synthase in tomato alters leaf carbohydrate partitioning. Plant Cell 3:1121-30 